\title{
Latent Heat Flux Profiles from Collocated Airborne Water Vapor and Wind Lidars during IHOP_2002
}

\author{
C. Kiemle, ${ }^{*}$ W. A. Brewer, ${ }^{+}$G. Ehret, ${ }^{*}$ R. M. Hardesty ${ }^{+}$A. FiX, ${ }^{*}$ C. SenfF, ${ }^{+}$M. Wirth, ${ }^{*}$ \\ G. PoberaJ,*,\# AND M. A. LeMone ${ }^{@}$ \\ * Institute of Atmospheric Physics, Deutsches Zentrum für Luft- und Raumfahrt (DLR), Oberpfaffenhofen, Germany \\ + Environmental Technical Laboratory, NOAA, Boulder, Colorado \\ @ Earth Observation Laboratory, NCAR, Boulder, Colorado
}

(Manuscript received 24 February 2006, in final form 30 May 2006)

\begin{abstract}
Latent heat flux profiles in the convective boundary layer (CBL) are obtained for the first time with the combination of the Deutsches Zentrum für Luft- und Raumfahrt (DLR) water vapor differential absorption lidar (DIAL) and the NOAA high resolution Doppler wind lidar (HRDL). Both instruments were integrated nadir viewing on board the DLR Falcon research aircraft during the International $\mathrm{H}_{2} \mathrm{O}$ Project (IHOP_2002) over the U.S. Southern Great Plains. Flux profiles from 300 to $2500 \mathrm{~m}$ AGL are computed from high spatial resolution ( $150 \mathrm{~m}$ horizontal and vertical) two-dimensional water vapor and vertical velocity lidar cross sections using the eddy covariance technique. Three flight segments on 7 June 2002 between 1000 and 1300 LT over western Oklahoma and southwestern Kansas are analyzed. On two segments with strong convection, the latent heat flux peaks at $(700 \pm 200) \mathrm{W} \mathrm{m}^{-2}$ in the entrainment zone and decreases linearly to $(200 \pm 100) \mathrm{W} \mathrm{m}^{-2}$ in the lower CBL. A water vapor budget analysis reveals that this flux divergence $\left[(0.9 \pm 0.4) \mathrm{g} \mathrm{kg}^{-1} \mathrm{~h}^{-1}\right]$ plus the advection $\left(0.3 \mathrm{~g} \mathrm{~kg}^{-1} \mathrm{~h}^{-1}\right)$ are nearly balanced by substantial CBL drying $\left[(1.5 \pm 0.2) \mathrm{g} \mathrm{kg}^{-1} \mathrm{~h}^{-1}\right]$ observed by airborne and surface in situ instruments, within the limits of the overall budget rms error of $0.5 \mathrm{~g} \mathrm{~kg}^{-1} \mathrm{~h}^{-1}$. Entrainment of dry air from aloft and net upward humidity transport caused the CBL drying and finally inhibited the initiation of deep convection. All cospectra show significant contributions to the flux between 1- and 10-km wavelength, with peaks between 2 and $6 \mathrm{~km}$, originating from large eddies. The main flux uncertainty is due to low sampling $(55 \% \mathrm{rmse}$ at mid-CBL), while instrument noise (15\%) and systematic errors ( $7 \%$ ) play a minor role. The combination of a water vapor and a wind lidar on an aircraft appears as an attractive new tool that allows measuring latent heat flux profiles from a single overflight of the investigated area.
\end{abstract}

\section{Introduction}

Water vapor is a relatively minor constituent of the earth's atmosphere but a major factor in atmospheric energetics and radiation, as well as transport and conversion of latent heat. Although the importance of water vapor is well recognized, its spatial and temporal variability is still poorly characterized by both observa-

\footnotetext{
\# Current affiliation: Institute for Quantum Electronics, Eidgenössische Technische Hochschule, Zürich, Switzerland.
}

Corresponding author address: Christoph Kiemle, Institute of Atmospheric Physics, Deutsches Zentrum für Luft- und Raumfahrt (DLR), Oberpfaffenhofen, 82230 Wessling, Germany.

E-mail: christoph.kiemle@dlr.de tions and models. While most of the horizontal humidity transport occurs through advection on large scales well resolved in atmospheric models, vertical transport is dominated by turbulence on subgrid-scale sizes and has to be parameterized. The transport of humidity through the CBL and into the free atmosphere above is a key element of the hydrological cycle. Water vapor and temperature profiles determine whether condensation at the CBL top, cloud growth, and occasionally deep convection with subsequent heavy precipitation will occur. Alternatively, entrainment of drier air from the free atmosphere may reduce CBL humidity and inhibit this sequence. This highlights the fundamental importance of the latent heat flux profile and its divergence (the vertical derivative of the flux), which is a crucial term in the CBL humidity budget.

Conventional latent heat flux profiling techniques

DOI: 10.1175/JTECH1997.1 
suffer from certain limitations. Tower in situ measurements sample only the lowest part of the CBL and are influenced by local upstream surface properties. Under low wind speed conditions the tower fluxes suffer from poor sampling and are biased by the CBL evolution. Better sampling is obtained by airborne instruments, but they are only available episodically for process studies because their high costs prevent them from being deployed operationally. Aircraft with in situ instruments have to fly the same pattern at different stacked altitudes in order to obtain a vertical flux profile, which considerably limits the operation range. The first remote sensing measurements of latent heat flux were ground-based and thus had to cope with similar problems as the towers, but with the advantage of a larger vertical range. Senff et al. (1994) and Wulfmeyer (1999) combined a zenith-pointing water vapor differential absorption lidar (DIAL) and a radio acoustic sounding system to obtain vertical profiles of the water vapor flux between 300-700 m AGL. Giez et al. (1999) used a Doppler lidar instead of the radar for the vertical velocity measurement and covered a range of 500-1300 m AGL. It was the first dual-lidar experiment for latent heat flux profiling.

Most limitations affecting ground-based and in situ instruments do not apply to airborne remote sensing. Flux profiles are obtained with great flexibility from a single overflight by water vapor and vertical velocity lidars installed on board the same aircraft. The test of new water vapor instruments was a main goal of the International $\mathrm{H}_{2} \mathrm{O}$ Project (IHOP_2002) field experiment that took place in May and June 2002 over the U.S. Southern Great Plains (Weckwerth et al. 2004). This campaign was, consequently, the ideal opportunity for the first collocated deployment of a water vapor and a wind lidar on aircraft. Substantial improvements of both the DLR water vapor DIAL and the National Oceanic and Atmospheric Administration (NOAA) high resolution Doppler wind lidar (HRDL) enabled the combined integration within the limited space and power availability of the DLR Falcon research aircraft. The lidars used two 40-cm-diameter aircraft bottom windows separated by $1 \mathrm{~m}$. With its long endurance and smooth in-flight behavior, the Falcon proved to be an excellent platform. Dropsondes and the University of Wyoming King Air aircraft provided data for validation. The paper is structured as follows. The description of the lidar systems and of the flux retrieval method is complemented by a detailed error analysis. The measurement results are discussed based on variance and flux profiles, spectra and cospectra. The case study concludes with an outlook.

\section{Method}

\section{a. The DLR water vapor DIAL}

Differential absorption lidar is a technique appropriate to remotely sense atmospheric trace gases like water vapor. The DIAL emits spectrally narrow and short laser pulses into the atmosphere at a wavelength tuned to the center of a water vapor molecular absorption line. Both the resulting online backscatter signals and the offline reference signals are used to compute the water vapor molecule number density as function of distance from the lidar. The offline backscatter signals also contain information on aerosols in the probed atmosphere. A DIAL installed on an airborne platform allows obtaining 2D backscatter and humidity cross sections from a single overflight. The DLR airborne water vapor DIAL participated in numerous field experiments and helped characterize the variability of water vapor and aerosols within the CBL and at its top (Kiemle et al. 1997). In 1997 a new system was developed, focusing on upper-tropospheric and lower-stratospheric water vapor (Ehret et al. 1999).

The system transmitter is based on an injectionseeded optical parametric oscillator (OPO) pumped by the second harmonic of a Q-switched, diode-pumped single-mode $\mathrm{Nd}$ :YAG laser at a repetition rate of 100 Hz. The OPO is optimized to operate in the spectral region between $920-950 \mathrm{~nm}$ at an average output power of up to $1.8 \mathrm{~W}$. The system performs simultaneous polarization-sensitive backscatter measurements at 532 and $1064 \mathrm{~nm}$ for aerosol characterization. In-flight quicklooks of aircraft and lidar data including twodimensional aerosol backscatter cross sections provide real-time information about the actual state of the system and the probed atmosphere. The system is designed to enable flexible operation with respect to wavelength selection. For IHOP_2002 with typically 4-km flight altitude and high ABL humidity a weak water vapor absorption line at $926.874 \mathrm{~nm}$ was selected with the help of a DIAL simulation program. A detailed description of the system and its quality is found in Poberaj et al. (2002).

To match the wind lidar spatial resolution the individual on- and offline DIAL profiles are accumulated to 1-s averages before the application of the DIAL equation, which is run at an effective vertical resolution of $150 \mathrm{~m}$. The typical aircraft speed of $150 \mathrm{~m} \mathrm{~s}^{-1}$ brings the horizontal resolution to be equal to the vertical resolution. No further filter is applied to the DIAL data. High data quality is ensured by rejecting all profiles with unclear or unstable laser behavior. The Falcon dropsondes' pressure and temperature profiles pro- 
TABLE 1. Systematic uncertainties ( $1 \sigma$ error) of water vapor DIAL measurements in the mid-CBL. References are (i) Behrendt et al. (2004) and (ii) Ismail and Browell (1989).

\begin{tabular}{lcc}
\hline \multicolumn{1}{c}{ Parameter } & Uncertainty & Reference \\
\hline $\mathrm{H}_{2} \mathrm{O}$ absorption cross section & $5 \%$ & (i) \\
Laser spectral purity & $3 \%$ & (i) \\
Atmospheric temperature variation & $4 \%$ & (i) \\
Atmospheric pressure variation & $0.3 \%$ & (i) \\
Rayleigh-Doppler effect & $0.5 \%$ & (ii) \\
Total (RMS) & $7.1 \%$ & \\
\hline
\end{tabular}

vide accurate means to translate from water vapor molecule number density into mass mixing ratio profiles. The IHOP_2002 DIAL data quality was recently assessed in extensive instrument intercomparison studies (Behrendt et al. 2004, 2007). Table 1 gives an overview of the most important systematic uncertainties resulting from these.

\section{b. The NOAA high resolution Doppler wind lidar}

The NOAA high resolution Doppler wind lidar (Grund et al. 2001) was deployed alongside the DLR water vapor DIAL on the DLR Falcon aircraft for IHOP_2002. HRDL uses heterodyne coherent detection to measure the radial component of the wind velocity by detecting the Doppler shift of radiation backscattered toward the lidar from atmospheric aerosols. Table 2 lists the main DIAL and HRDL system parameters. For IHOP_2002, HRDL was deployed in one of two configurations. To measure horizontal water vapor transport, a refractive wedge was inserted above the aircraft optical window to deflect the beam $15^{\circ}$ off nadir. The beam was fixed perpendicular to the aircraft flight direction, providing a single component of the wind. To maximize the impact of the single component measurements, the aircraft flew box patterns, whereby the primary component of the southerly low-level jet in the central Plains was measured directly and the water vapor transport characterized and quantified (Hardesty et al. 2003).

For the measurements of vertical velocity and water vapor flux profiles presented in this paper, the lidar pointed nadir to estimate vertical velocities. To ensure exact nadir pointing, adjustments were undertaken after each aircraft turn at the flight leg ends. In addition, the vertical velocity data were corrected for residual biases due to short-term aircraft motions in between the adjustment phases by using the ground return as reference. The systematic uncertainty is hence much lower than for the DIAL. The wind lidar returns were processed to yield a horizontal resolution of $1 \mathrm{~s}(150 \mathrm{~m})$ and a vertical resolution of $150 \mathrm{~m}$. For quality control, the HRDL signal-to-noise ratio (SNR) is stored for
TABLE 2. DIAL and HRDL system parameters.

\begin{tabular}{lll}
\hline \multicolumn{1}{c}{ Parameter } & \multicolumn{1}{c}{ DIAL } & \multicolumn{1}{c}{ HRDL } \\
\hline Transmitter type & OPO & Diode laser \\
Wavelength $(\mathrm{nm})$ & 927 & 2020 \\
Pulse energy $(\mathrm{mJ})$ & 12 & 2 \\
PRF (Hz) & 100 & 200 \\
Pulse length (ns) & 7 & 200 \\
Detection principle & Direct & Heterodyne \\
Detector type & APD & PIN diode \\
Telescope diameter $(\mathrm{cm})$ & 35 & 20 \\
Horizontal resolution $(\mathrm{m})^{\mathrm{c}}$ & 1.5 & 0.75 \\
Vertical resolution $(\mathrm{m})^{\mathrm{c}}$ & 15 & 30 \\
Absolute accuracy & $0.6 \mathrm{~g} \mathrm{~kg}^{-1, \mathrm{~d}}$ & $0.05 \mathrm{~m} \mathrm{~s}^{-1, \mathrm{e}}$ \\
\hline
\end{tabular}

${ }^{\text {a }}$ Avalanche Photo Diode (APD).

${ }^{\mathrm{b}}$ Positive-intrinsic-negative zone (PIN).

${ }^{\mathrm{c}}$ Resolution of the unprocessed raw data.

${ }^{\mathrm{d}}$ Total from Table 1 for a water vapor mixing ratio of $8.5 \mathrm{~g} \mathrm{~kg}^{-1}$.

${ }^{\mathrm{e}}$ Residual uncertainty when using the ground return as zero vertical wind speed reference.

each data point. Tests revealed that already a small number of erroneous spikes (data outliers) distort the HRDL spectra. They are interspersed among the data whenever there is not enough aerosol backscatter. Then the SNR falls below a critical threshold value. This is the case in roughly one-fifth of the whole dataset. In these areas a running $2 \times 2$ median filter is applied to the two-dimensional velocity field: each of the data points with bad SNR is replaced by the median value of its 4-pixel neighborhood. This method very efficiently removes scattered outliers while preserving most of the high spatial resolution. The few remaining spikes are removed individually.

\section{c. Computing fluxes with the eddy covariance method}

Turbulent diffusion is the dominating mechanism for the vertical transport of trace gases in the CBL. Turbulence is generated by buoyant eddies with mid-CBL horizontal extent of up to several times the CBL depth. Near the surface additional turbulence is caused by frictional drag on smaller scales. In the entrainment zone, the interfacial layer between the well-mixed CBL and the free atmosphere, turbulence can be created by wind shear and breaking gravity waves. It is yet unclear which turbulent transport mechanism dominates in the entrainment zone on average. In fact, the different turbulence generation mechanisms acting in different places, on different scales, interacting with each other and dependent on various boundary conditions make CBL turbulence so complex.

Trace gas transport is quantified by the flux, defined as the net mass transfer of a quantity per unit area per unit time. The turbulent vertical water vapor flux at a 
given height is equal to the covariance between the vertical velocity $w\left(\mathrm{~m} \mathrm{~s}^{-1}\right)$ and water vapor mixing ratio $q\left(\mathrm{~g} \mathrm{~kg}^{-1}\right)$ time series. The flux covariance is analytically identical to the integral of the cospectrum of $w$ and $q$, defined as the real part of the cross spectrum between $w$ and $q$ (cf. e.g., Stull 1988). Hence, the flux can be computed either from the covariance or from the cospectrum of $w$ and $q$. Both ways were tested in the present study, yielding identical results. A vertical profile of the flux is obtained from the two-dimensional $w$ and $q$ lidar cross sections by computing the covariance of each horizontal $w$ and $q$ series, separated in the vertical by $150 \mathrm{~m}$ according to the vertical resolution of the lidar data. Since no extra vertical averaging is applied, this equals the vertical resolution of the flux profile. To get rid of undersampled mesoscale contributions to the flux and in order to cope adequately with data gaps caused by laser adjustments, the $w$ and $q$ time series are cut into individual segments of $80 \mathrm{~s}(12 \mathrm{~km})$ length each. They are positioned such as to avoid data gaps and are overlapping by one-half of their length wherever possible. This yields the smallest variance in the spectra (Press et al. 1988).

For each segment, the mean, variance and covariance profiles are determined and spectra are computed by applying an FFT on the time series of both $w$ and $q$ after quality control and subtraction of the mean value. Finally, the statistics and spectra are accumulated over all segments of a flight leg. This method considerably improves the statistical significance and is superior to conventional filtering techniques because it can bypass data gaps. The water vapor flux $\left(\mathrm{g} \mathrm{kg}^{-1} \mathrm{~m} \mathrm{~s}^{-1}\right)$ is translated into the flux of latent heat $\left(\mathrm{W} \mathrm{m}^{-2}\right)$ by multiplication with the air density $\rho$ and the latent heat of vaporization of water $L_{v}$. Both $\rho$ and $L_{v}$ increase with decreasing temperature and thus partly compensate the decrease of $\rho$ with height in a vertical CBL profile of the product $\rho L_{v}$. In the flux profiles presented here, $\rho L_{v}$ at the CBL top height is only $5 \%$ smaller than at the surface. It is therefore convenient to use a constant product $\rho L_{v}$ that in the present case amounts to $2.4 \mathrm{MJ}$ $\mathrm{m}^{-3}$. The CBL temperature and water vapor fluctuations impinge on the dry air density. This leads to small deviations of the flux when trace gas densities are measured as is always the case with DIAL. Webb et al. (1980) presented a detailed derivation of first-order flux corrections. Assuming equality between dry and moist air densities, which is correct to within $\sim 99 \%$, we can rewrite their relationship for the latent heat flux $F$ as:

$$
F_{\text {corr }}=\left(1+\frac{M_{\mathrm{air}}}{M_{\mathrm{H} 2 \mathrm{O}}} \bar{q}\right)\left(F+F_{\mathrm{SH}} \frac{L_{v}}{c_{p}} \frac{\bar{q}}{\bar{T}}\right) .
$$

The molar mass ratio of dry air and water $M_{\text {air }} / M_{\mathrm{H} 2 \mathrm{O}}$ being 1.60, the first bracket yields 1.01 for a mean water vapor mixing ratio of $0.008\left(8 \mathrm{~g} \mathrm{~kg}^{-1}\right)$. In the second bracket $F_{\mathrm{SH}}$ is the sensible heat flux and $c_{p}$ the specific heat of dry air at constant pressure. The correction becomes important for large Bowen ratios $\left(F_{\mathrm{SH}} \gg F\right)$, which is not the case in the present study. The dimensionless factor associated to $F_{\mathrm{SH}}$ amounts to approximately 0.06 here. Assuming a sensible heat flux at the surface of $300 \mathrm{~W} \mathrm{~m}^{-2}$ (from flux tower measurements) and a linear decrease of $F_{\mathrm{SH}}$ with height (from CBL theory), the Webb-corrected latent heat flux is approximately $3 \%$ larger than the uncorrected flux at mid-CBL and $1 \%$ larger at the CBL top for our flux profiles. Because the flux measurement uncertainties are more than an order of magnitude larger, we did not apply the Webb correction.

\section{Measurement uncertainties}

A thorough assessment of all potentially important errors is mandatory for flux measurements because covariances are particularly sensitive to sensor misalignments, instrumental noise, and bad or missing data. For instance, data gaps that have the size of the dominant turbulent scales cannot simply be bridged by interpolation or zero padding because these methods disturb the phase information of the atmospheric signature and may bias the resulting flux. For this reason, an outstanding data quality is required, implying data with low noise and bias, and with gaps smaller in size than the dominant turbulent scales. This requires the absence of CBL top clouds that would cause lidar data gaps of critical size. Unfortunately, the dataset was additionally limited due to system failures from thermal overload in the aircraft cabin. All constraints reduced the available amount of collocated IHOP_2002 water vapor and wind lidar measurements to one good flight. This section demonstrates that even in this case a couple of important uncertainties persist. They are summarized in Table 3.

\section{a. Sampling uncertainty}

The most important uncertainty in the present study arises from the fact that the measurement area is limited in size; that is, only a restricted number of fluxgenerating eddies can be sampled. This is also the largest uncertainty in comparable studies (Senff et al. 1994; Giez et al. 1999; Wulfmeyer 1999). The sampling error is related to the ratio of the flux integral scale $\lambda_{F}$ and the flight leg length $L$ as follows (Lenschow and Stankov 1986):

$$
\sigma_{F, \text { sampling }}^{2}=2 \frac{\lambda_{F}}{L}\left(\sigma_{w}^{2} \sigma_{q}^{2}+F^{2}\right) .
$$


TABLE 3. Flux measurement uncertainties for flight legs 3 and 4 at mid-CBL. The Webb correction, deviation from ensemble mean, collocation, and pointing offsets are considered small enough to be neglected.

\begin{tabular}{llc}
\hline \hline Flux uncertainty & Main reason & $\begin{array}{c}\text { Mid-CBL } \\
\text { values }\end{array}$ \\
\hline Sampling & Flight leg length & $55 \%$ \\
Instrument noise & DIAL noise & $15 \%$ \\
Instrument bias & See Table 1 & $7 \%$ \\
Webb correction & Air density fluctuations & $3 \%$ \\
Deviation from ensemble & Finite time series & $0.6 \%$ \\
$\quad$ mean & Lag between $w$ and $q$ & $<75 \mathrm{~m}$ \\
Collocation offset & DIAL telescope & $<45 \mathrm{~m}$ \\
Pointing offset & mounting tolerance & \\
& & $58 \%$ \\
Total (RMS)* & &
\end{tabular}

* Sampling, instrument noise, and instrument bias uncertainties geometrically added.

This expression yields the absolute sampling error; $\sigma_{w}^{2}$ and $\sigma_{q}^{2}$ are the vertical velocity and water vapor mixing ratio variances. The fluxes in the present study have relative sampling errors between $25 \%$ and $55 \%$. Since the flux-dominating eddies are found to extend vertically throughout most of the CBL, the sampling error cannot be reduced by vertical averaging of the flux profile.

\section{b. Instrument noise}

Instrument noise is the second important measurement uncertainty in the present study. Lidar measurements contain uncorrelated noise from, for example, the random nature of the scattering process or the transmitter and detector photon statistics. Giez et al. (1999) present an appropriate representation of the instrumental noise uncertainty:

$$
\begin{aligned}
\sigma_{F, \text { noise }}^{2}= & \frac{\Delta L}{L}\left(\sigma_{w}^{2} \sigma_{q, \text { noise }}^{2}+\sigma_{w, \text { noise }}^{2} \sigma_{q}^{2}\right. \\
& \left.+\sigma_{w, \text { noise }}^{2} \sigma_{q, \text { noise }}^{2}\right)
\end{aligned}
$$

where $\Delta L$ is the horizontal spacing between two measurements (here $150 \mathrm{~m}$ ) and $\sigma_{w, \text { noise }}^{2}$ and $\sigma_{q \text {,noise }}^{2}$ are the $w$ and $q$ variances from instrumental noise. The fluxes in the present study have relative noise errors between $4 \%$ and $30 \%$. The lowest errors are found in the near range at the CBL top. While the wind lidar data are nearly noise-free because of the heterodyne detection principle, the DIAL data are contaminated with noise that increases nonlinearly with range. Since the noise is uncorrelated, averaging reduces it by the square root of the number of averaged data points. As with the sampling error, an increase of the flight leg length also reduces the noise. Alternatively, an increase of the measurement frequency (decreasing $\Delta L$ ) would help, but this is limited by the instruments' performance.

\section{c. Finite series deviation from ensemble mean}

The fact that the measurement time series have finite length generates a deviation from the ensemble average flux. Lenschow et al. (1994) derived the following expression for this systematic flux error:

$$
\frac{F-\langle F(L)\rangle}{F} \approx 2 \frac{\lambda_{F}}{L} .
$$

In the present study this expression gives values between $0.4 \%$ and $0.9 \%$, and the flux deviation can therefore be neglected when compared to the sampling and noise uncertainties.

\section{d. Collocation and pointing offsets}

The telescopes of both lidar systems were separated by about $1 \mathrm{~m}$ in flight direction. This is negligible given the horizontal resolution of $150 \mathrm{~m}$. However, both systems run independently so that data timing had to be checked carefully. Thanks to GPS time and position stored by both systems the time lag uncertainty between DIAL and HRDL is confined to within acceptable bounds of half a time step $(0.5 \mathrm{~s}$ or $75 \mathrm{~m})$.

While the HRDL beam was pointed nadir, this position being regularly checked and eventually corrected, the DIAL beam was constantly perpendicular to the aircraft horizontal axis due to mechanical constraints. This results in a DIAL off-nadir pointing equal to the aircraft pitch angle (the angle between the horizontal aircraft axis and the horizon) of $4.5^{\circ}$. The angular offset between both beams is corrected by a range-dependent shift of the DIAL time series relative to the HRDL data. The aircraft pitch and roll angle variations are negligibly small $\left(< \pm 0.2^{\circ}\right)$ and only straight flight legs are exploited so that the roll angle is zero on average. The DIAL pointing has an approximately $1^{\circ}$ residual uncertainty due to telescope mounting tolerances. This translates into a maximum $45-\mathrm{m}$ horizontal lag at the end of the measurement range ( $300 \mathrm{~m} \mathrm{AGL}$ ) which can be neglected. The collocation and pointing offsets were double-checked by cross correlating the ground return altitude measured independently by both systems. Occasionally, cloud return signals could be used for the same purpose. This also permitted to check the vertical collocation with an accuracy of half a range cell (75 m). 


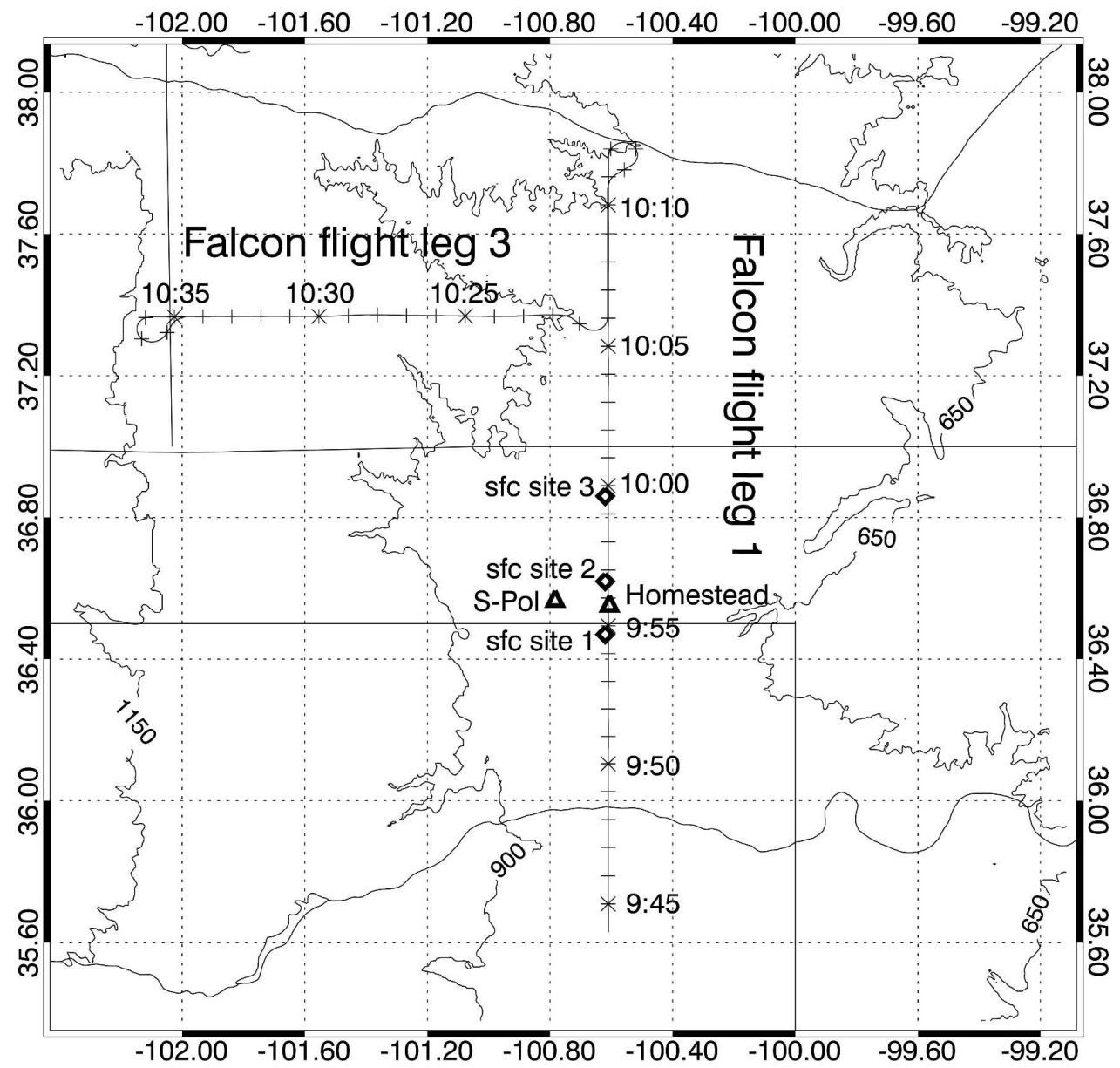

FIG. 1. Map showing DLR Falcon aircraft track, NCAR surface flux sites, and surface elevation contours (m MSL). For better clarity only two flight legs are plotted. Leg 4 was flown on the track of leg 3 , and leg 9 on the track of leg 1 .

\section{Results and discussion}

\section{a. Measurements overview}

On 7 June 2002, a CBL heterogeneity mission was on the IHOP_2002 agenda. The weather was dominated by a high pressure system with moderate low-level southerly winds. The flights were made over semiarid, partially artificially irrigated flat terrain interspersed with creeks. The mean ground altitude for the flight legs 1 and 9 was $860 \mathrm{~m}$ and for the legs 3 and 4 it was $1000 \mathrm{~m}$ MSL. Figure 1 gives an overview of the aircraft operations. In situ data for comparison were provided by the University of Wyoming King Air aircraft. While the King Air's objective was more the study of the CBL evolution within a small area, the DLR Falcon's mission was to map a larger domain by flying a crossshaped pattern with a size of about $300 \mathrm{~km}$. The King Air aircraft flew 13 short $(54 \mathrm{~km})$ north-south-oriented legs between $36.5^{\circ}$ and $36.9^{\circ} \mathrm{N}$ at $100.6^{\circ} \mathrm{W}$ along the
IHOP_2002 western track in alternating altitudes of $60 \mathrm{~m} \mathrm{AGL}$ and $0.7 z_{i}$ with interspersed vertical soundings. Because of lidar data gaps, only the four Falcon flight legs indicated in Fig. 1 could be analyzed. Legs 1 and 9 were separated in time by about $3 \mathrm{~h}$, located above the King Air track, while the legs 3 and 4 were more to the west, and flown roughly half an hour later than leg 1.

The King Air aircraft measured winds with a Rosemount 858AJ/1332 differential pressure gust-probe system. Position and motion relative to the ground were measured by a Honeywell Laseref SM inertial navigation system with low-frequency altitude based on the pressure altitude. The aircraft location was corrected using GPS to within $100 \mathrm{~m}$. Humidity was measured by a Lyman alpha hygrometer referenced to a chilledmirror hygrometer. Variances and fluxes were processed without filtering after removal of the linear trend for each leg. The surface water vapor mixing ra- 


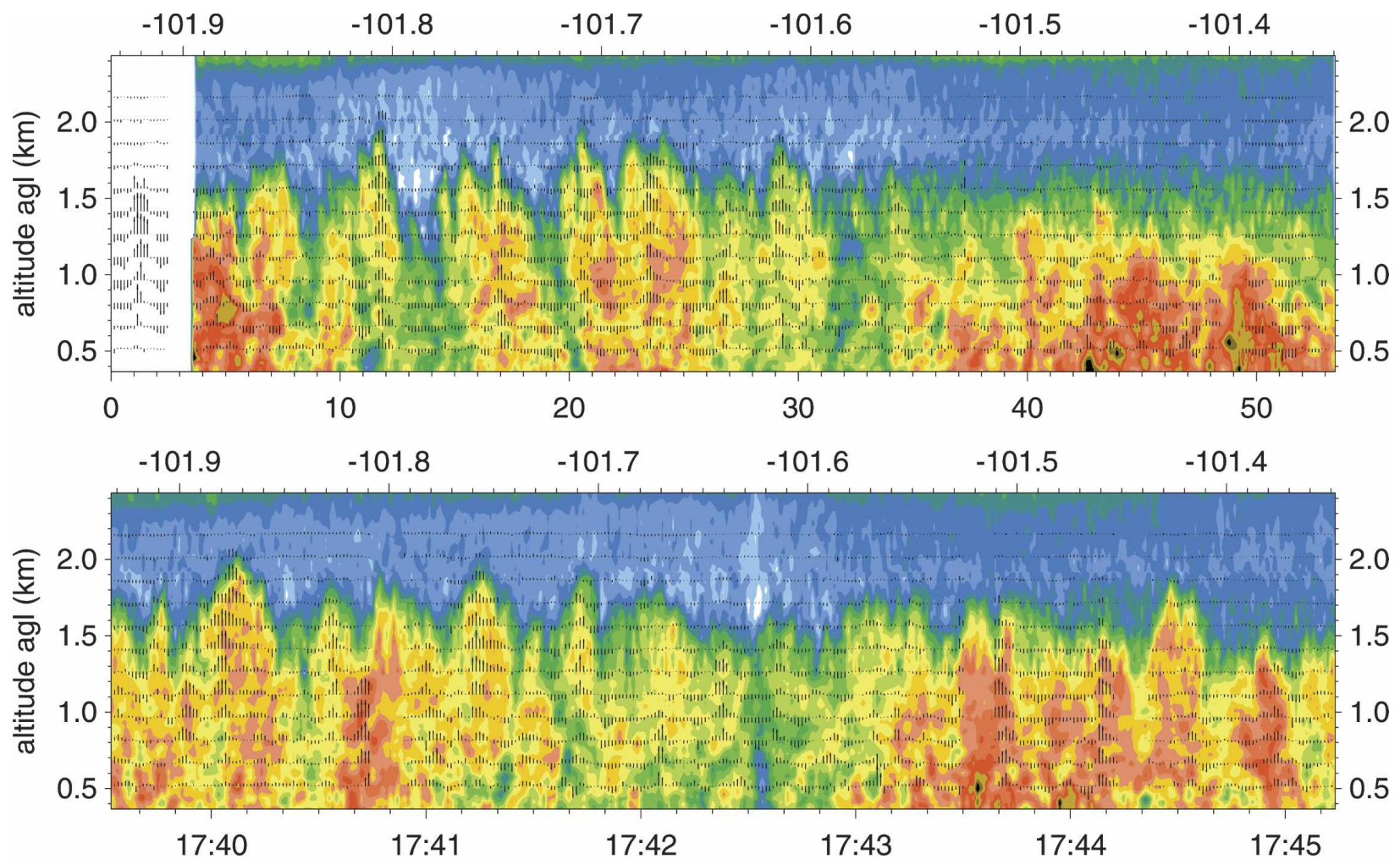

DLR DIAL Water Vapour Mixing Ratio and NOAA HRDL Wind Velocity on 7.6.02., Legs 3 and 4

$$
\begin{array}{llllllllllllllll}
3.0 & 3.5 & 4.0 & 4.5 & 5.0 & 5.5 & 6.0 & 6.5 & 7.0 & 7.5 & 8.0 & 8.5 & 9.0 & 9.5 & 10.0 & \mathrm{~g} / \mathrm{kg}
\end{array}
$$

FIG. 2. Vertical cross sections of water vapor (colors) and vertical velocity (arrows) for the Falcon flight legs 3 and 4 oriented west-east at $37.4^{\circ} \mathrm{N}$ over southwestern Kansas. The aircraft flew (top) leg 3 from right (east) to left (west) and turned back at $102^{\circ} \mathrm{W}$ to (bottom) fly leg 4 on the same track. Top axis is longitude, bottom axis distance (km), and UTC time, $7 \mathrm{~h}$ ahead of LT. Maximum vertical wind velocities are $-4.2 \mathrm{~m} \mathrm{~s}^{-1}$ in downward and $6.6 \mathrm{~m} \mathrm{~s}^{-1}$ in upward directions. An arrow length corresponding to 150-m altitude difference is $7 \mathrm{~m} \mathrm{~s}^{-1}$ in vertical velocity. The aspect ratio is about $1: 7$; that is, the cross sections are compressed horizontally by a factor of 7 . It is evident that strong contributions to the flux emanate from the largest thermals.

tios were measured at $2-3 \mathrm{~m} \mathrm{AGL}$ at three National Center for Atmospheric Research (NCAR) surfaceflux stations located along the IHOP_2002 western track, using a Vaisala 50Y Hummitter (http://www.rap. ucar.edu/research/land/observations/ihop.php). Site 1 (bare ground) was near the south end of the track, site 2 (ungrazed grass) near the center of the track, and site 3 (sagebrush) near the north end of the track. The surface fluxes were computed as half-hour averages.

Figure 2 displays the superposition of vertical velocity and water vapor lidar measurements for parts of flight leg 3 (top) and 4 (bottom). The aircraft turned back at $102 \mathrm{~W}$; hence, the time interval between the two legs was $6 \mathrm{~min}$ at the left edge and $17 \mathrm{~min}$ at the right edge of Fig. 2. Horizontal CBL winds as measured by a Falcon dropsonde in the middle of leg 4 were $15 \mathrm{~m} \mathrm{~s}^{-1}$ from south-southwest (SSW), that is, nearly perpen- dicular into the plane of the cross sections. This explains the absence of comparable structures in both plots. The measurements stop at $300 \mathrm{~m}$ AGL because of the vertical range cell size of $150 \mathrm{~m}$ for both lidars and slight ground-level variations. The superposition of $w$ and $q$ gives evidence of considerable correlation between elongated humidity structures and upward motion. Strong contributions to the water vapor transport quantified by the vertical flux emanate obviously from the largest thermals that are found to extend vertically throughout most of the CBL. An inactive old thermal is visible in leg 4 at $101.42^{\circ} \mathrm{W}$, in contrast. There is also evidence of very deep entrainment, for example, in leg 4 at $101.62^{\circ} \mathrm{W}$, where dry air is transported downward.

Figure 2 shows that CBL top determination is difficult, if not impossible, for one-dimensional profilers such as radio- or dropsondes. The humidity gradient 
TABLE 4. Main characteristics of the flight legs and overview of the results.

\begin{tabular}{|c|c|c|c|c|c|}
\hline Description & Symbol & Unit & Flight leg 1 & Legs 3 and 4 & Leg 9 \\
\hline See figure & & & Fig. 6a & Figs. $2,3,4,6 b$ & Fig. 6c \\
\hline LT (UTC - 7 h) & & & 0945-1010 & $1023-1045$ & $1238-1253$ \\
\hline Position & & & $35.7^{\circ}-37.7^{\circ} \mathrm{N}, 100.6^{\circ} \mathrm{W}$ & $37.4^{\circ} \mathrm{N}, 100.9^{\circ}-101.9^{\circ} \mathrm{W}$ & $35.5^{\circ}-36.8^{\circ} \mathrm{N}, 100.6^{\circ} \mathrm{W}$ \\
\hline Flight leg length & $L$ & $\mathrm{~km}$ & 123 & 121 & 86 \\
\hline No. of 80 -s segments & & & 14 & 18 & 10 \\
\hline CBL top height & $z_{i}$ & $\mathrm{~m}$ & $690 \pm 230$ & $1240 \pm 320$ & $1560 \pm 220$ \\
\hline $\begin{array}{l}\text { Vertical velocity integral length } \\
\text { scale }\end{array}$ & $\lambda_{w}$ & $\mathrm{~m}$ & $400 \pm 100\left(0.6 z_{i}\right)$ & $350 \pm 50\left(0.3 z_{i}\right)$ & $400 \pm 50\left(0.3 z_{i}\right)$ \\
\hline Water vapor integral length scale & $\lambda_{q}$ & $\mathrm{~m}$ & $800 \pm 200\left(1.2 z_{i}\right)$ & $800 \pm 200\left(0.7 z_{i}\right)$ & $700 \pm 200\left(0.5 z_{i}\right)$ \\
\hline $\begin{array}{l}\text { Flux integral length scale (within } \\
\text { CBL) }\end{array}$ & $\lambda_{\mathrm{wq}}$ & $\mathrm{m}$ & $250 \pm 50\left(0.4 z_{i}\right)$ & $370 \pm 50\left(0.3 z_{i}\right)$ & $400 \pm 200\left(0.3 z_{i}\right)$ \\
\hline Cospectrum zero at & & $\mathrm{m}$ & $1000\left(1.5 z_{i}\right)$ & $1000\left(0.8 z_{i}\right)$ & $1700\left(1.1 z_{i}\right)$ \\
\hline Cospectrum maximum & & $\mathrm{m}$ & $2300\left(3.3 z_{i}\right)$ & $4000\left(3.2 z_{i}\right)$ & $6000\left(3.9 z_{i}\right)$ \\
\hline Max $q$ variance & $\sigma_{q, \max }^{2}$ & $\left(\mathrm{~g} \mathrm{~kg}^{-1}\right)^{2}$ & 0.5 & 0.7 & 0.9 \\
\hline Max $w$ variance & $\sigma_{w, \max }^{2}$ & $\left(\mathrm{~m} \mathrm{~s}^{-1}\right)^{2}$ & 0.8 & 2.0 & 1.5 \\
\hline Max flux & $F_{\max }$ & $\mathrm{W} \mathrm{m}^{-2}$ & $400 \pm 120$ & $780 \pm 210$ & $650 \pm 250$ \\
\hline CBL flux divergence & $d F / d z$ & $\mathrm{~g} \mathrm{~kg}^{-1} \mathrm{~h}^{-1}$ & - & $0.90 \pm 0.26$ & $0.86 \pm 0.41$ \\
\hline
\end{tabular}

toward the much drier free atmosphere identifies the irregular CBL top. The DIAL aerosol backscatter data exhibit a similar structure (high aerosol load in the $\mathrm{CBL}$, cleaner above) at higher spatial resolution. We apply the wavelet analysis technique from Davis et al. (2000) to find the instantaneous CBL top in each individual lidar backscatter profile. A statistical analysis yields the mean CBL top height (denoted $z_{i}$ ) and its standard deviation for each flight leg; the results are shown in Table 4 and in Figs. 3 and 6. The mean heights agree with locations of the halfway jump in humidity between the CBL and the free atmosphere in legaveraged water vapor DIAL profiles.

\section{b. Variance profiles, integral scales, and power spectra}

The total measured variance is the sum of pure atmospheric and instrument noise variances. While the nearly noise-free wind lidar data are only contaminated by scattered spikes that are easy to detect and remove, the DIAL data are affected by white noise that increases with range. Since the instrument noise of both DIAL and HRDL lidars is neither correlated with atmospheric structures nor with each other, it is superposed as noise peak to the pure atmospheric variances $\sigma_{q}^{2}$ and $\sigma_{w}^{2}$ in the horizontal autocovariance functions' zero lag and can be separated using, for example, the method described in Kiemle et al. (1997). They use a linear fit through the first three lags of the autocovariance function, extrapolated to the zero lag, in order to get a robust estimate of atmospheric variances not contaminated by instrument noise. The method is applied in the present study to the $w$ and $q$ data. At a horizontal resolution of $150 \mathrm{~m}$, the fit extends across $450 \mathrm{~m}$, which corresponds roughly to the observed $w$ integral length scale.

Alternative methods, including fits that are more sophisticated or the use of the power spectrum in order to separate atmospheric and noise variances, were described by Lenschow et al. (2000). The power spectrum approach consists of using the high-frequency part of the spectrum where the signal is completely overwhelmed by noise, assuming the noise is white. The noise variance is estimated as the average level of the spectrum's high-frequency part. This approach is difficult to apply to airborne data because of the limited horizontal resolution that leaves a very narrow or inexistent white noise region at the high-frequency end of the spectrum. Lenschow et al. (2000) also investigate a more elaborate method to extrapolate to the zero lag in the autocovariance function, which they find to be in very close agreement with their power spectrum results. Instead of a linear fit through the first lags of the autocovariance function they use a $2 / 3$ power-law fit suggested by a model of the inertial subrange. We tested this method but rejected it because our horizontal and vertical resolution of $150 \mathrm{~m}$ is coarser by a factor $2-5$ compared to Lenschow et al. (2000). We do not sample all of the small-scale atmospheric variability and cannot apply the proposed nonlinear fit because our autocovariance functions are too coarse. This is subject for further investigations with future airborne sensors that will have higher spatial resolution.

Figure 3 shows profiles of water vapor and vertical velocity variance for the flight legs 3 and 4 . The difference between pure atmospheric and measured variance 


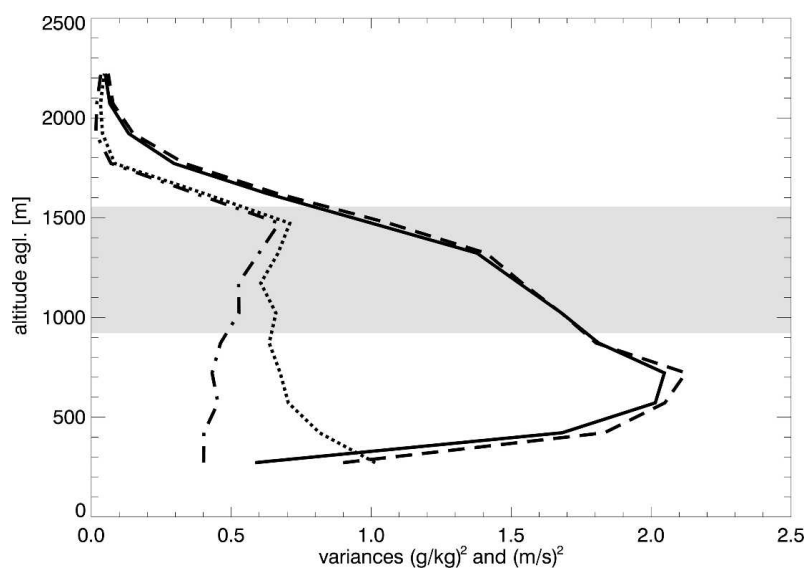

FIG. 3. Profiles of pure atmospheric variance for vertical velocity (solid) and water vapor (dash-dotted) for the flight legs 3 and 4. The dashed and dotted curves represent the corresponding measured variances including instrumental noise. The CBL top variability [mean $\left(z_{i}\right) \pm \operatorname{std} \operatorname{dev}\left(z_{i}\right)$ ] derived from lidar backscatter profiles is expressed by the gray area. Maximum variances are as expected located at the CBL top for water vapor and at midCBL for vertical velocity.

is the uncorrelated instrumental noise that strongly increases with range for the DIAL while being nearly absent in the HRDL data. Maximum $q$ variances, also for the other flight legs not shown here, are located at the CBL top as consequence of the strong water vapor gradient between the CBL and the free atmosphere, visible in Fig. 2. The maximum vertical velocity variances for all flight legs are found at mid-CBL. This is in agreement with large eddy simulations (Moeng and Wyngaard 1989) and observations (Lenschow et al. 2000). It has to do with the fact that the strongest and largest buoyant eddies predominate in the mid-CBL while the entrainment zone and the surface layer contain eddies with smaller spatial scales and less turbulent energy.

Integral length scales are deduced from the autocovariance function using the method described in Lenschow and Stankov (1986); that is, the function is integrated between lag one and the first zero crossing. The integration result is normalized by the atmospheric variance. To estimate the flux sampling error [Eq. (2)] the integral scale of the flux is needed. It is determined in similar way by integrating the cross-covariance function of $w$ and $q$ between the zero lag and the first zero crossing, and normalizing with the zero lag value. This is possible because there is no correlation between the DIAL and HRDL instrument noise; hence, the zero lag of the cross-covariance function is not contaminated by a noise peak. Rather, all cross-covariance functions within the CBL exhibit a distinct atmospheric maximum around the zero lag, related to positive fluxes. The

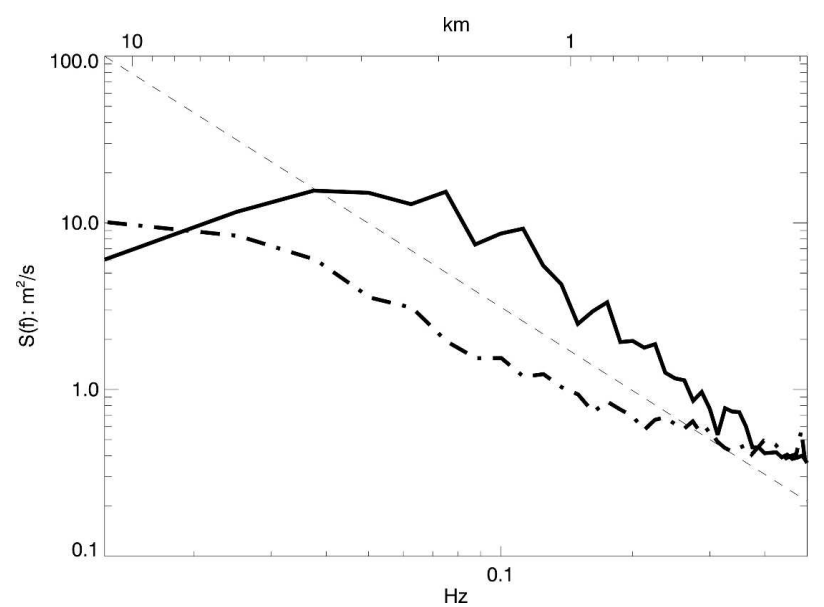

FIG. 4. Power spectra of vertical velocity (solid) and water vapor mixing ratio (dash-dotted) for the flight legs 3 and 4, vertically averaged over seven individual spectra between $400 \mathrm{~m}\left(0.3 z_{i}\right)$ and $1300 \mathrm{~m}\left(z_{i}\right)$ AGL. The dashed line is a $-5 / 3$ power-law slope of the inertial subrange for comparison. The upper scale represents wavelength of atmospheric structures, the lower scale frequency. The well-pronounced vertical velocity spectral peak between 2and 4-km wavelength (1.6 to $3.2 z_{i}$ ) stems from the largest turbulence-generating thermals. In contrast, the water vapor spectrum exhibits a smooth transition to the mesoscale.

resulting integral scales are listed in Table 4 with their corresponding variation ranges within the CBL. Compared to the data from Lenschow and Stankov (1986), our flux integral scales are larger by a factor of 2 to 3 . This may be because we are observing a situation with vigorous convection over land; their measurements were mainly over the ocean. In addition, Fig. 2 shows that the high variability of the local CBL height makes normalizing by $z_{i}$ somewhat arbitrary. We conclude that we have not enough measurements for a solid statement in this respect.

Figure 4 presents $w$ and $q$ power spectra for legs 3 and 4 from a vertical average of seven individual spectra between $0.3 z_{i}$ and $z_{i}$. The vertical average reduces instrumental noise in the spectra. It does, however, not much improve the sampling uncertainty at low frequency because the atmospheric structures are highly correlated in the vertical. The well-pronounced peak in the vertical velocity spectrum between 2 - and $4-\mathrm{km}$ wavelength (1.6 to $3.2 z_{i}$ ) stems from the largest turbulence-generating thermals. Unlike the $w$ spectrum the $q$ spectrum does not show a peak but rather a smooth transition toward the mesoscale. This is expected as scalars and the horizontal velocity components typically show no reduction in amplitude at long wavelengths in contrast to the vertical velocity whose scales are constrained by the surface and the capping inversion (D. Lenschow 2005, personal communication). 


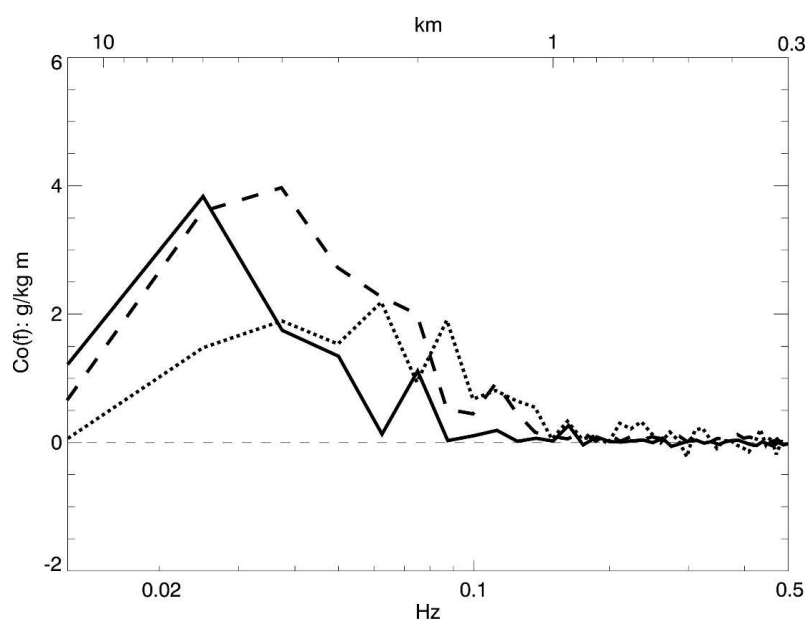

FIG. 5. Cospectra of vertical velocity and water vapor mixing ratio for flight legs 1 (dotted), 3 and 4 (dashed), and 9 (solid). The dotted line represents the cospectrum of leg 1 at $0.6 z_{i}$, while the dashed and solid lines represent vertical averages of seven individual cospectra between $0.3 z_{i}$ and $z_{i}$. The main contributions to the latent heat flux stem from scales that span roughly an order of magnitude, from 1 to $10 \mathrm{~km}$, peaking between 2- and 6-km wavelength. There is no contribution from scales smaller than $1 \mathrm{~km}(1.7$ $\mathrm{km}$ with leg 9).

\section{c. Cospectra of vertical velocity and water vapor}

Figure 5 shows cospectra for all the three independent flux measurements. Cospectra contain the contributions of $w$ and $q$ that are in phase and unfold the spatial turbulent scales responsible for the flux. Like the spectra, the cospectra were averaged vertically between about $0.3 z_{i}$ and $z_{i}$ in order to reduce noise, except in leg 1 where the CBL is less developed and where the cospectral amplitudes are roughly half as large. The slight increase of the dominant scales in leg 9 is attributed to the fact that leg 9 was flown 2 to $3 \mathrm{~h}$ later, shortly after local noon. We find little difference with regard to the dominating spatial scales between the three cospectra. All contributions to the latent heat flux are from scales that span roughly an order of magnitude, between 1 and $10 \mathrm{~km}$, with peaks between 2 and $6 \mathrm{~km}$ wavelength, which corresponds to 3 to $4 z_{i}$ depending on the flight leg (cf. Table 4).

All cospectra reveal that there is no contribution to the flux from scales smaller than $1 \mathrm{~km}$. This is consistent with the results of former work (Senff et al. 1994; Giez et al. 1999; Wulfmeyer 1999), with the difference that the cospectra of the present work are less noisy, thanks to improved instrumentation. It is also consistent with cospectra from the King Air aircraft upper-level $\left(0.7 z_{i}\right)$ legs flown at about the same time and place as the Falcon's leg 9. Furthermore, the scales of the lidar and in situ cospectrum maxima agree. Note that in contrast the low-level (60 m AGL) King Air cospectra do con- tain significant flux contributions at scales smaller than $1 \mathrm{~km}$. We find that large thermals are responsible for most of the flux in the mid- and upper CBL, while water vapor in the lower CBL is transported vertically on smaller scales. This is in agreement with previous work dedicated to understand the complex nature of turbulent transport and to identify the flux dominating structures. Duncan and Schuepp (1992), for example, find that isolating individual structures linked to physical transport phenomena such as eddies or thermals reveals that a few structures are responsible for most of the flux. However, former studies suffered from a high level of instrumental noise or bad sampling conditions. This paper demonstrates that now there is the possibility to investigate turbulent transport in detail throughout the CBL with the use of accurate and low-noise cospectra.

\section{d. Latent heat fluxes}

The water vapor flux profiles in Fig. 6 were derived using the method described in section $2 \mathrm{c}$ and present the principal results of the study. In all figures, the flux is zero in the free atmosphere above the tops of the strongest thermals, as expected. In Fig. 6a, the CBL is in an earlier stage with less vigorous convection as indicated by the lower CBL top and the $w$ variances of Table 4. Figures $6 b, c$ show a quasi-linear flux decrease to $200 \mathrm{~W} \mathrm{~m}^{-2}$ at $0.35 z_{i}$. The flux profile of Fig. $6 \mathrm{c}$ is noisier because of higher instrumental noise and a shorter flight leg that increases the sampling uncertainty. Nevertheless, a strong positive flux divergence is consistently apparent in Figs. 6b,c. It represents a significant upward humidity transport drying out the midand lower CBL by entrainment of dry air from above (see also Fig. 2), while the entrainment zone and the part of the free atmosphere that is penetrated by the strongest thermals are humidified.

The overlaid sampling and noise uncertainty profiles characterize the quality of the flux measurements. They show that the sampling error is nearly constant with height within the CBL and that the instrumental noise increases with range from the lidar. An important quality check is, of course, the validation by other instruments, if available and applicable. Fluxes were measured in situ by the University of Wyoming King Air aircraft and NCAR surface flux towers. Unfortunately, due to the different aircraft mission objectives on that day there were only two intercomparison opportunities with the King Air at $0.6 z_{i}$ (in Fig. 6c), and the towers as well as the low-level King Air legs were $\sim 300 \mathrm{~m}$ below the lowest lidar flux measurements. No in situ measurements are available in the east-west track of Fig. 6b. Figures 6a,c show that the King Air in situ 
fluxes are larger than the surface and the lidar measured fluxes. The King Air flight legs are $54 \mathrm{~km}$ long and cover only the central part of the lidar domain (cf. Fig. 1). In addition, the King Air fluxes are computed from unfiltered time series, whereas the lidar fluxes represent averages over multiple segments. Unfiltered
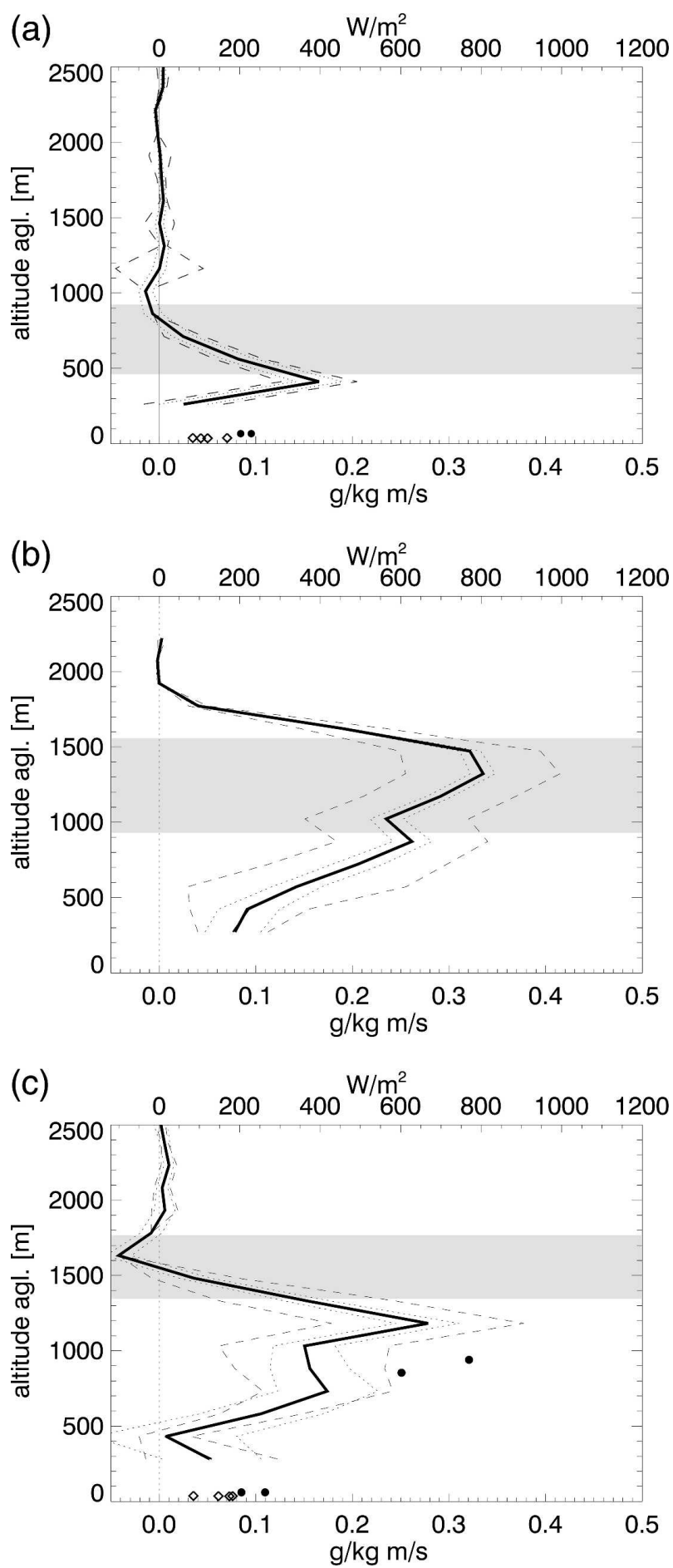

mesoscale contributions may account for the observed higher King Air values, but the lack of additional measurements inhibits a robust statement to this respect. We conclude that within the frame of this limited analysis, the lidar fluxes agree fairly well with the in situ fluxes. For future campaigns, we recommend that high priority be given to instrument intercomparison efforts since it is an excellent means for quality control.

\section{e. Water vapor budget analysis}

Under the assumption that the water vapor mixing ratio is conserved; that is, does not undergo phase changes (e.g., cloud condensation), the simplified budget equation for $q$ within a turbulent flow, neglecting the horizontal flux divergence, relates the temporal change of $q$ to the advection and to the vertical flux divergence (e.g., Stull 1988):

$$
\frac{d \bar{q}}{d t}+\bar{u} \frac{d \bar{q}}{d x}+\bar{v} \frac{d \bar{q}}{d y}+\frac{d \overline{w^{\prime} q^{\prime}}}{d z}=0
$$

The vertical flux divergence of water vapor is estimated from the lidar flux profiles, whereby the profile is linear under the assumptions of quasi-steady CBL conditions and vertically uniform advection. A weighted linear fit to the profile of Fig. $6 \mathrm{~b}$ between 300 and $1300 \mathrm{~m}$ yields a flux divergence of $(0.90 \pm 0.26) \mathrm{g}$ $\mathrm{kg}^{-1} \mathrm{~h}^{-1}$; we obtain $(0.86 \pm 0.41) \mathrm{g} \mathrm{kg}^{-1} \mathrm{~h}^{-1}$ for the profile of Fig. 6c (cf. Table 4). The sampling and noise uncertainty profiles are used as fit weights so that the fit also delivers the flux divergence uncertainty. On four stacked flight legs at $60 \mathrm{~m}$ AGL and $0.6 z_{i}$ between 1200 and $1300 \mathrm{LT}$ a linear fit to the King Air covariances

FIG. 6. (a) Lidar-derived water vapor flux profile for the northsouth-oriented leg 1 (solid; around 1000 LT) with sampling uncertainty [thin dashed; after Eq. (2)] and instrumental noise [thin dotted; after Eq. (3)]. Top axis is the latent heat flux. The large CBL top variation range is expressed by the gray area. The filled circles represent the University of Wyoming King Air in situ fluxes from two $60 \mathrm{~m}$ AGL flight legs. The diamonds are half-hour averages from four NCAR integrated surface flux facility towers (for locations, see Fig. 1). (b) Flux profile for the east-west-eastoriented legs 3 and 4 (solid; around 1030 LT), with uncertainty ranges as in (a). The flux peaks at the CBL top and is zero in the free atmosphere above the tops of the strongest thermals. The strong positive flux divergence is associated with a significant upward humidity transport drying out the mid- and lower CBL by deep entrainment of dry air and humidifying the free atmosphere as the CBL grows vertically. (c) Flux profile for the north-south leg 9 around 1245 LT with in situ fluxes as in (a). Both a shorter flight leg and higher instrumental noise make the profile noisier in comparison to (b). A similar flux divergence indicates ongoing CBL drying shortly after local noon. 
gives a flux divergence of $(0.8 \pm 0.6) \mathrm{g} \mathrm{kg}^{-1} \mathrm{~h}^{-1}$ which is in close agreement with the lidar results. These King Air measurements are coincident in time and space with the Falcon leg 9 and are shown in Fig. 6c.

Significant CBL drying was observed in situ by the NCAR surface flux towers and the King Air. It amounts to $(1.53 \pm 0.24) \mathrm{g} \mathrm{kg}^{-1} \mathrm{~h}^{-1}$ between 1200 and 1300 LT for three surface stations and two low-level King Air flight legs that were closest to the Falcon's leg 9. The uncertainty is the standard deviation between the individual $d q / d t$ gradients, which are computed from half-hour averages of the surface time series and from flight leg averages of the aircraft. CBL drying is expected from Eq. (5) to balance the positive flux divergence measured, unless advection dominates. In Eq. (5), the advection term is split into its two horizontal components. The King Air and the Falcon dropsondes measured moderate winds with $10 \mathrm{~m} \mathrm{~s}^{-1}$ from SSW throughout the CBL without significant altitude dependence. The mean (and standard deviation) wind over four King Air flight legs at $60 \mathrm{~m} \mathrm{AGL}$ and $0.6 z_{i}$ between 1200 and $1300 \mathrm{LT}$ is $(1.4 \pm 0.6) \mathrm{m} \mathrm{s}^{-1}$ for the $u$ and $(9.7 \pm 1.2) \mathrm{m} \mathrm{s}^{-1}$ for the $v$ wind component. The Oklahoma Mesonet data reveal a pronounced westeast humidity gradient $d q / d x$ of $-0.015 \mathrm{~g} \mathrm{~kg}^{-1} \mathrm{~km}^{-1}$ over the area with less humidity to the west as expected. The south-north gradient $d q / d y$ is weaker $\left(0.008 \mathrm{~g} \mathrm{~kg}^{-1}\right.$ $\mathrm{km}^{-1}$ ) with more humidity to the south as observed by the NCAR surface stations. Since $u$ and $d q / d y$ are small, both advection terms are small although $v$ and $d q / d x$ are relatively large. Introducing all above results valid between 1200 and $1300 \mathrm{LT}$, between $35.5^{\circ}$ and $36.8^{\circ} \mathrm{N}$ at $100.6^{\circ} \mathrm{W}$, into Eq. (5) yields

$$
[(-1.53 \pm 0.24)+(-0.08 \pm 0.04)+(0.38 \pm 0.17)+(0.86 \pm 0.41)] \mathrm{g} \mathrm{kg}^{-1} \mathrm{~h}^{-1}=(-0.37 \pm 0.51) \mathrm{g} \mathrm{kg}^{-1} \mathrm{~h}^{-1}
$$

The right-hand side's deviation from zero represents an imbalance of $-0.37 \mathrm{~g} \mathrm{~kg}^{-1} \mathrm{~h}^{-1}$ smaller than the overall rmse uncertainty of $0.51 \mathrm{~g} \mathrm{~kg}^{-1} \mathrm{~h}^{-1}$ composed of the geometric sum over all uncertainties of the lefthand side of Eq. (6). This result can be interpreted as a successful closure of the water vapor budget equation, given the fact that aside from the measurement uncertainties, a couple of assumptions and simplifications led to Eq. (6). The assumed vertical uniformity of $q$ advection and horizontal gradients, and CBL stationary conditions are to a certain extent questionable. Because of both limited instrumental coverage and high complexity with large $q$ gradients and CBL depth variability, it is impossible to obtain a more precise handle of the situation. However, we find that the instrumental and methodological uncertainties do not limit the value of the case study.

\section{Conclusions}

A water vapor DIAL and a Doppler wind lidar were installed for the first time on board an aircraft in order to measure CBL flux profiles via eddy correlation. On 7 June 2002, a meteorologically relevant situation was observed above the U.S. Great Plains. The lidar measurements around local noon reveal a pronounced flux divergence linked to a large entrainment flux. A detailed instrument error assessment and a humidity budget analysis show that instrumental and methodological uncertainties somewhat limit the accuracy, but not the value of the approach, despite the limited dataset. The observed substantial CBL drying by net upward humidity transport and entrainment of dry air from aloft finally inhibited the growth of clouds and deep convection. The case study can be seen as a crosscheck to convection initiation. This makes it important in the frame of actual efforts to improve the forecast of heavy precipitation events. The prediction skill of warm season convective precipitation is poor, despite advances in the quality of numerical weather prediction models and increased water vapor data assimilation efforts. This is mainly because the models must parameterize convection as a subgrid-scale process and because current water vapor measurements are inaccurate and incomplete (Weckwerth et al. 2004). Here, the described new instrumentation may fill in a gap in the future, at least for process studies. The next deployment of a water vapor and a wind lidar on the Falcon aircraft is planned during the Convective and Orographicallyinduced Precipitation Study (COPS; www.unihohenheim.de/spp-iop/index.htm) in southwestern Germany in 2007 aimed at improving precipitation forecasts in complex terrain.

Acknowledgments. The participation of the DLR Falcon in IHOP_2002 was sponsored by NSF and ESA. Our thanks are extended to the DLR flight facility. Useful comments from Don Lenschow and Ken Davis helped concentrating on the most relevant topics. 


\section{REFERENCES}

Behrendt, A., C. Kiemle, P. Di Girolamo, H.-S. Bauer, T. Schaberl, and V. Wulfmeyer, 2004: Measurement and intercomparison of active, passive, and in situ sensors during the International $\mathrm{H}_{2} \mathrm{O}$ Project for the verification of the specifications of the WALES experiment. ESA Final Rep. 16669/02/ $\mathrm{NL} / \mathrm{FF}, 56 \mathrm{pp}$.

_ , and Coauthors, 2007: Intercomparison of water vapor data measured with lidar during IHOP_2002. Part I: Airborne to ground-based lidar systems and comparisons with chilledmirror radiosondes. J. Atmos. Oceanic Technol., 24, 3-21.

Davis, K. J., N. Gamage, C. Hagelberg, D. H. Lenschow, C. Kiemle, and P. P. Sullivan, 2000: An objective method for deriving atmospheric structure from airborne lidar observations. J. Atmos. Oceanic Technol., 17, 1455-1468.

Duncan, M. R., and P. H. Schuepp, 1992: A method to delineate extreme structures within airborne flux traces over the FIFE site. J. Geophys. Res., 97, 18 487-18 498.

Ehret, G., K. P. Hoinka, J. Stein, A. Fix, C. Kiemle, and G. Poberaj, 1999: Low-stratospheric water vapor measured by an airborne DIAL. J. Geophys. Res., 104, 31 351-31 359.

Giez, A., G. Ehret, R. L. Schwiesow, K. J. Davis, and D. H. Lenschow, 1999: Water vapor flux measurements from groundbased vertically pointed water vapor differential absorption and Doppler lidars. J. Atmos. Oceanic Technol., 16, 237-250.

Grund, C. J., M. Banta, J. George, J. Howell, M. Post, R. Richter, and A. Weickmann, 2001: High-resolution Doppler lidar for boundary layer research. J. Atmos. Oceanic Technol., 18, 376-393.

Hardesty, R. M., and Coauthors, 2003: Airborne measurements of horizontal wind and moisture transport using co-deployed Doppler and DIAL lidars. Proc. Int. Symp. on Tropospheric Profiling, Leipzig, Germany, Institute for Tropospheric Research and German Weather Service, 390-392.

Ismail, S., and E. V. Browell, 1989: Airborne and spaceborne lidar measurements of water vapor profiles: A sensitivity analysis. Appl. Opt., 28, 3603-3614.
Kiemle, C., G. Ehret, A. Giez, K. J. Davis, D. H. Lenschow, and S. P. Oncley, 1997: Estimation of boundary-layer humidity fluxes and statistics from airborne differential absorption lidar (DIAL). J. Geophys. Res., 102, 29 189-29 203.

Lenschow, D. H., and B. B. Stankov, 1986: Length scales in the convective boundary layer. J. Atmos. Sci., 43, 1198-1209.

_ J. Mann, and L. Kristensen, 1994: How long is long enough when measuring fluxes and other turbulence statistics? J. Atmos. Oceanic Technol., 11, 661-673.

-, V. Wulfmeyer, and C. Senff, 2000: Measuring secondthrough fourth-order moments in noisy data. J. Atmos. Sci., 17, 1330-1347.

Moeng, C.-H., and J. C. Wyngaard, 1989: Evaluation of turbulent transport and dissipation closures in second-order modeling. J. Atmos. Sci., 46, 2311-2330.

Poberaj, G., A. Fix, A. Assion, M. Wirth, C. Kiemle, and G. Ehret, 2002: Airborne all-solid-state dial for water vapour measurements in the tropopause region: System description and assessment of accuracy. Appl. Phys. B, 75, 165-172.

Press, W. H., B. P. Flannery, S. A. Teukolsky, and W. T. Vetterling, 1988: Numerical Recipes in C: The Art of Scientific Computing. Cambridge University Press, $735 \mathrm{pp}$.

Senff, C., J. Bösenberg, and G. Peters, 1994: Measurement of water vapor flux profiles in the convective boundary layer with lidar and radar-RASS. J. Atmos. Oceanic Technol., 11, 85-93.

Stull, R. B., 1988: An Introduction to Boundary Layer Meteorology. Kluwer Academic, 645 pp.

Webb, E. K., G. I. Pearman, and R. Leuning, 1980: Correction of flux measurements for density effects due to heat and water vapour transfer. Quart. J. Roy. Meteor. Soc., 106, 85-100.

Weckwerth, T. M., and Coauthors, 2004: An overview of the International $\mathrm{H}_{2} \mathrm{O}$ Project (IHOP_2002) and some preliminary highlights. Bull. Amer. Meteor. Soc., 85, 253-277.

Wulfmeyer, V., 1999: Investigations of turbulent processes in the lower troposphere with water vapor DIAL and radar-RASS. J. Atmos. Sci., 56, 1055-1076. 